\title{
Nuclear transport inhibition in acute myeloid leukemia: recent advances and future perspectives
}

\author{
Chetasi Talati ${ }^{1} \&$ Kendra L Sweet ${ }^{*, 1}$ \\ ${ }^{1}$ Malignant Hematology, H. Lee Moffitt Cancer Center \& Research Institute, Tampa, Florida, 33611, USA \\ *Author for correspondence: Kendra.sweet@moffit.org
}

\section{Practice points}

- Novel therapeutic approaches for treatment of acute myeloid leukemia are urgently needed.

- XPO1 inhibitors represent an emerging class of novel agents that alter the nuclear transport of various proteins including many tumor suppressor proteins and others. Examples include p53, NPM1, NFk $\beta$ that are important for regulation of cell cycle propagation.

- First generation SINE, selinexor, has demonstrated clinical efficacy in early phase clinical trials with a tolerable adverse event profile including fatigue, nausea, anorexia and myelosuppression.

- KPT-8602 is a promising, highly potent, second-generation SINE, with improved tolerance due to lack of CNS penetration in patient-derived xenograft mouse models.

- Strategies that incorporate SINEs with conventional chemotherapy are promising and further investigations focusing on specific molecular abnormalities should be explored.

Selectiveinhibitors of nuclear export (SINE) are emerging as a potentially efficacious therapeutic strategy for overcoming resistance to conventional chemotherapy for acute myeloid leukemia. SINE specifically block the protein Exportin 1, also known as chromosomal region maintenance 1, leading to nuclear retention of cargo proteins, including several tumor suppressor proteins. Selinexor, a first generation SINE, is currently in early phase clinical studies in various combinations with promising antileukemic and proapoptotic activity. Here we discuss the mechanism of action of SINEs and further elaborate on the clinical data available from the various trials in acute myeloid leukemia.

First draft submitted: 15 February 2018; Accepted for publication: 7 June 2018; Published online: 11 September 2018

Keywords: acute myeloid leukemia • CRM1 inhibitor • KPT-8602 • nuclear transport inhibitor • selinexor • XPO1 inhibitor

Acute myeloid leukemia (AML) is a heterogeneous disease characterized by expansion of clonal myeloid precursors in the bone marrow and blood. Unfortunately, little improvement has been made in long-term outcomes for AML patients, in whom the 5-year overall survival (OS) is estimated at 26.9\% [1]. Standard induction therapy for AML has remained largely unchanged over several decades. Induction chemotherapy with an anthracycline and cytarabine is considered a standard therapeutic approach for patients who are able to tolerate an intensive approach to treatment. Furthermore, the addition of other cytotoxic agents such as thioguanine, fludarabine, cladribine or etoposide to the backbone of standard induction therapy with anthracycline and cytarabine has not translated into meaningful improvements in the overall outcomes $[2,3]$. Although intensification of the anthracycline dose has improved survival in younger patients, the same benefit was not observed in older patients or those with adverse cytogenetics [4,5]. More recently, new drugs such as midostaurin and CPX-351 have been shown to improve survival in FLT3-mutated patients or patients with secondary AML, respectively, however even with these new treatment options, the majority of AML patients ultimately relapse and succumb to their disease [6,7]. Outcomes are, of course, particularly poor in those with secondary AML or adverse-risk cytogenetics [8,9]. With increased understanding of the pathways that allow for leukemia stem cell survival, along with their potential as therapeutic targets, new approaches are being investigated that have the potential to improve overall outcomes.

Future Medicine 


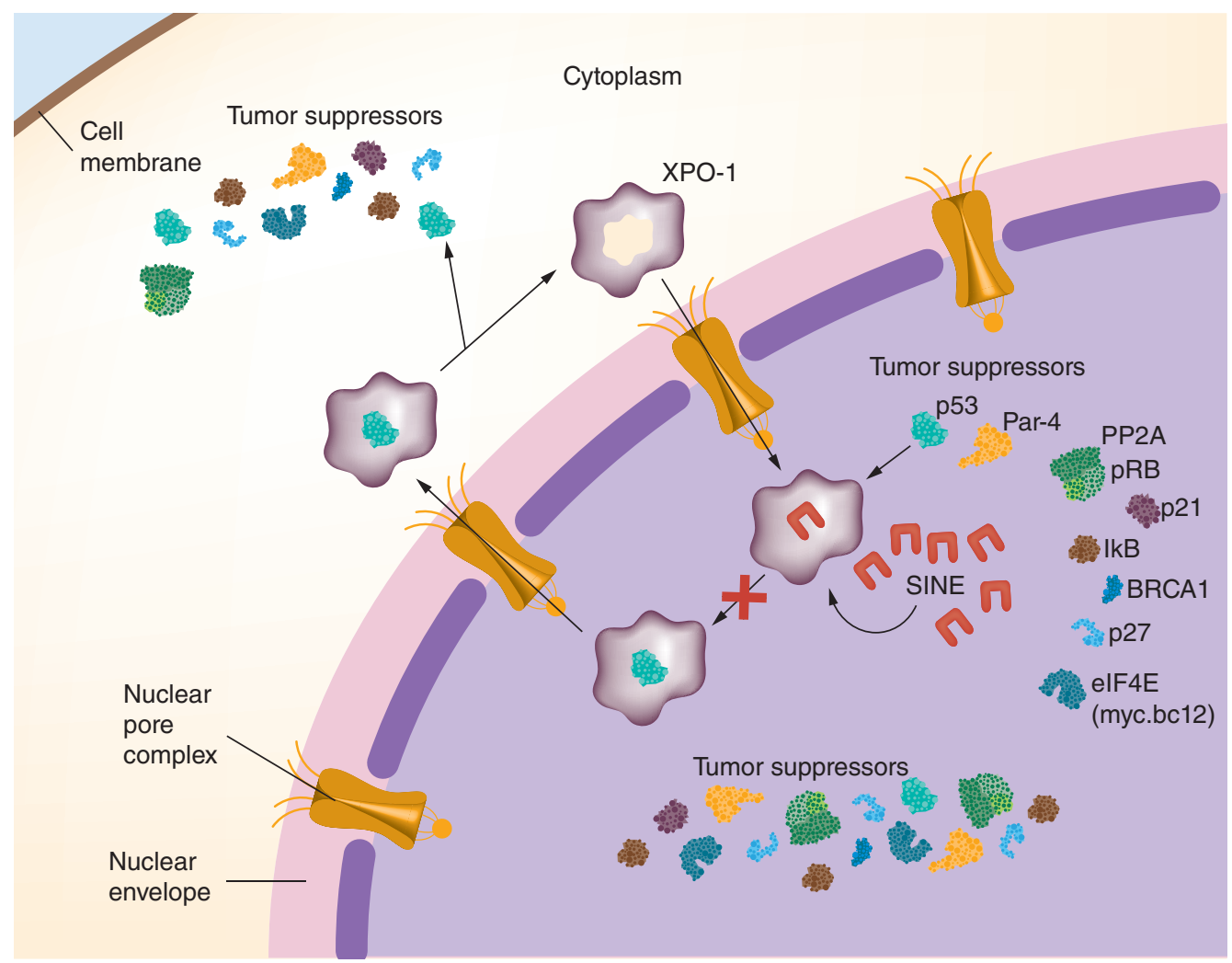

Figure 1. Mechanism of action of selective inhibitors of nuclear export.

\section{Role of exportin-1 in AML}

Exportin-1 (XPO1), also known as chromosome maintenance protein 1 (CRM1), is a nuclear export receptor involved in the cytoplasmic translocation of tumor suppressor proteins (TSPs) including p53, p21, p27, p73, nucleophosmin-1 (NPM1), protein phosphatase 2, forkhead box protein O3 (FOXO1), $\beta$-catenin/antigenpresenting cell, topoisomerase II and NF- $\kappa \beta$ /inhibitory nuclear factor $\kappa \beta[10,11]$. Nuclear export of TSPs leads to inactivation of these proteins and allows the malignant cells to evade apoptosis. Kojima et al. have demonstrated that increased expression of XPO1 is associated with higher risk AML and shorter OS [12]. Using the reverse phase protein array on 511 newly diagnosed AML patients, levels of XPO1 were profiled to assess prognostic impact. Higher levels of XPO1 were significantly correlated with higher marrow blast percentages, white blood cell counts, peripheral blood blast percentages and absolute peripheral blood blast count. In patients with favorable risk cytogenetics, expression of XPO1 was significantly lower compared with those with intermediate risk or adverse-risk cytogenetics $(p=0.029)$. Moreover, previous studies have found that higher expression of XPO1 is associated with AML with FMS-like tyrosine kinase 3 (FLT3) mutations which is a known adverse molecular finding [12-14]. Additionally, increased XPO1 levels were identified as an independent negative predictor of OS in a multivariate analysis. In patients with high XPO1 expression, the median OS was only 37 weeks, compared with 66 weeks in patients with low XPO1 expression $(\mathrm{p}=0.007)$ [12]. Forcing nuclear retention of TSPs will theoretically restore the normal function of these proteins and serves as a rational therapeutic strategy in AML.

\section{Selective inhibitors of nuclear export: mode of action \& preclinical studies}

Selective inhibitors of nuclear export (SINE) are small molecules that prevent the shuttling of intranuclear TSPs into the cytoplasm. In turn, this restores the functionality of TSPs allowing for maximum proapoptotic and antileukemic activity [15]. These novel, orally bioavailable compounds (referred to from here onwards as KPT-SINE), specifically and irreversibly bind to XPO1, thereby blocking its function as a nuclear export protein. Binding of KPT-SINE to the cysteine ${ }^{528}$ residue of XPO1 alters the protein conformation such that it is unable to bind TSPs, subsequently blocking their normal efflux from the nucleus (Figure 1). Ranganathan $e t$ al. initially demonstrated that treatment with KPT-SINE results in cell cycle arrest at the G1 phase, along with a concomitant decrease of cells in $S$ phase and 
G2/M phase. As such, this blocks the proliferation of AML blasts resulting in apoptosis [16]. Moreover, KPT-SINE reduces the level of functional XPO1 expression which contributes to greater intranuclear accumulation of XPO1 cargo proteins. In AML xenograft mouse models, treatment with KPT-SINE resulted in a reduction of the overall leukemic burden and significantly prolonged survival [16].

The antileukemic effect of XPO1 inhibitors was apparent across various genotypes and molecular subgroups. Kojima et al. demonstrated induction of full length p53 protein and p53-dependent proapoptotic activity [12]. Cells from AML patients exhibited lower sensitivity to KPT-SINE if they harbored TP53 mutations compared with the wild-type TP53 protein suggesting that an intact TP53 protein is required for an antileukemic effect. It has been well documented that cytoplasmic accumulation of nucleophosmin 1 (NPM1) occurs in NPM1-mutated $\left(\mathrm{NPM}^{\mathrm{mut}}\right)$ AML $[11,16]$. With inhibition of XPO1, NPM1 ${ }^{\text {mut }}$ is retained in the nucleus rather than being exported into the cytoplasm. The $\mathrm{IC}_{50}$ of KPT-SINE is lower in cytogenetically normal AML patients who also harbor a NPM1 ${ }^{\text {mut }}$, indicating higher vulnerability of this specific population to XPO1 inhibitors [16]. Treatment of AML cell lines and AML patient samples with KPT-SINE decreased the level of XPO1 protein and led to intranuclear accumulation of the XPO1 cargo proteins, specifically p53 and NPM1, allowing for restoration of their normal cellular function [16]. Post-transcriptional changes caused by KPT-SINE also led to a robust down-regulation of the total FLT3 protein level and the oncogenic c-Kit protein in both AML cell lines and primary AML patient samples irrespective of the mutational status $[12,16]$.

Another mechanism by which KPT-SINE leads to reduced leukemic burden is by promoting differentiation of leukemic blasts. This has been shown in preclinical studies with AML cell lines where investigators noted a significant increase in the expression of the cell surface myeloid differentiation marker, CD11b, after treatment with KPT-SINE. Along with the flow cytometric assessment, morphologic evaluation of the AML blasts also revealed changes consistent with differentiation; namely increased granulation and chromatin condensation [16].

Ranganathan and colleagues have explored the synergism of KPT-SINE and cytotoxic chemotherapy given the interaction between topoisomerase (Topo) II and XPO1 [16]. Mutations in Topo II $\alpha$ lead to the localization of this enzyme in the cytoplasm resulting in resistance to Topo II inhibitors in lung cancer. Although such mechanisms of resistance have not specifically been explored in AML, it is known that the export of Topo II is XPO1 dependent [1719]. Functionally, Topo II inhibitors such as anthracyclines induce DNA cleavage complexes and cell death. This function is disrupted when the enzyme is exported out of the nucleus. To this end, Ranganathan et al. evaluated the combinatorial effect of KPT-SINE (namely selinexor, discussed below) and Topo II inhibitors (idarubicin, etoposide, mitoxantrone and daunorubicin) in AML cell lines and primary AML patient samples [20]. Combination treatment not only restored the Topo II $\alpha$ to the nucleus, but also led to down-regulation of the DNA damage repair proteins Chk1 and Rad51, as well as mismatch repair proteins MSH2, MLH1, PMS2 and MSH6, ultimately leading to apoptosis. Moreover, levels of c-Myc, which is a positive regulator of Rad51 and Chk1, are greatly reduced in AML cell lines after treatment with selinexor.

Given the broad mechanisms of antileukemic activity, along with its functionality across various cytogenetic and molecular genotypes, KPT-SINE represents a rational and promising therapeutic option in AML. Several clinical trials have explored the efficacy of KPT-SINE in AML. The most well-studied compound to date is selinexor (KPT-330), which has been used as a single agent and in combination with various cytotoxic agents in multiple early phase clinical trials. On the basis of synergism between KPT-SINE and Topo II inhibitors, several clinical trials have combined selinexor with topo II inhibitors and other DNA damaging agents and will be described below [20-22].

\section{Selinexor (KPT-330) in clinical studies}

Selinexor (KPT-330) is a first generation, orally bioavailable, SINE that has been extensively studied in multiple early phase clinical trials in AML (Table 1). Selinexor has been used as a single agent and in combination with cytotoxic chemotherapy in previously untreated AML and relapsed or refractory AML patients.

\section{Selinexor in treatment-naive adverse risk AML}

A Phase I clinical trial with selinexor in combination with daunorubicin and cytarabine $(7+3)$ enrolled 21 patients with previously untreated, adverse risk AML, and demonstrated encouraging activity (NCT02403310) [21]. The median age of patients enrolled was 68 years, and $43 \%$ of patients were $\geq 70$ years of age. Early results were presented at the American Society of Hematology Annual Meeting in 2016. The primary end point of the study was the maximal tolerated dose (MTD) and recommended Phase II dose (RP2D) of selinexor. The MTD was not 


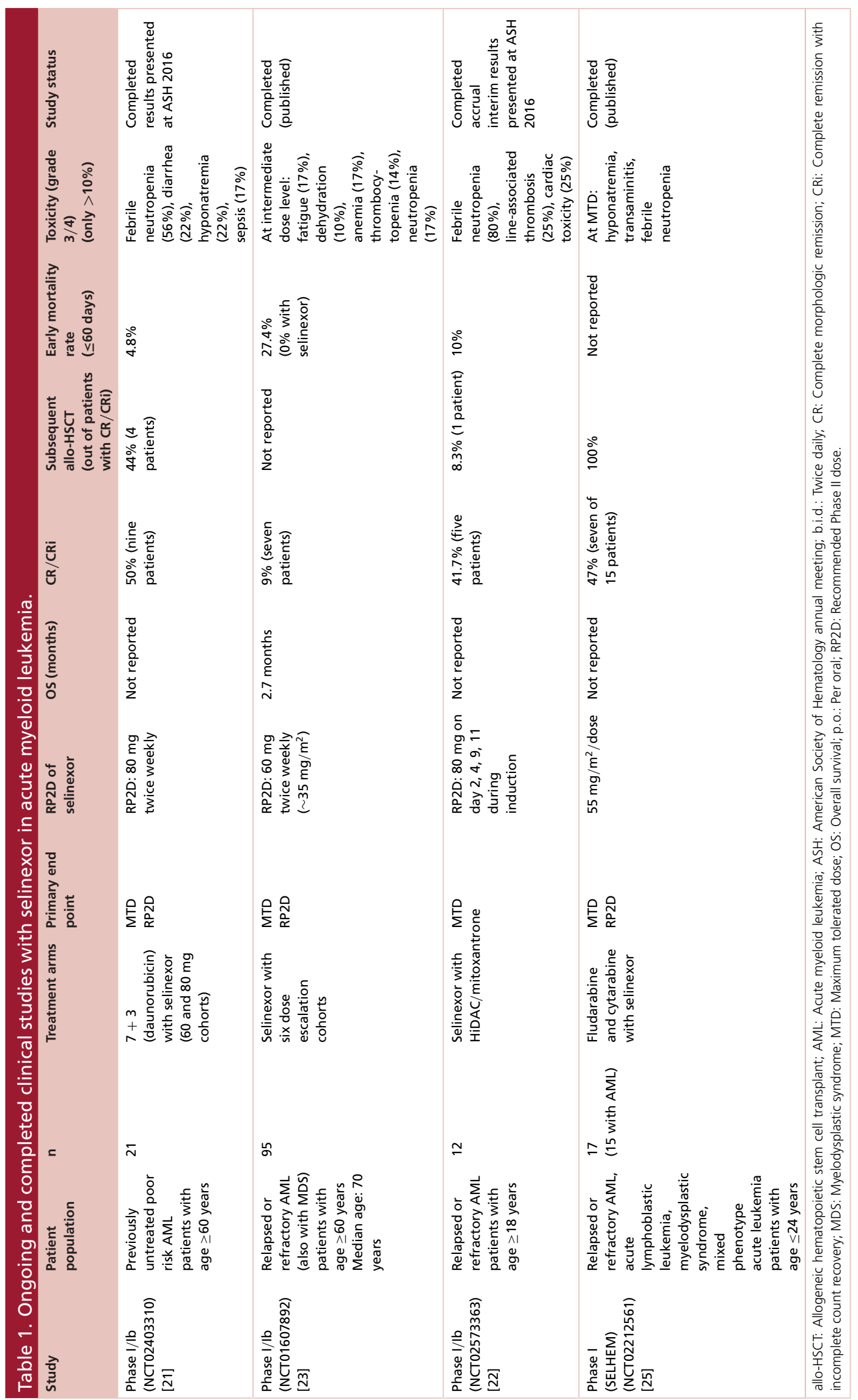




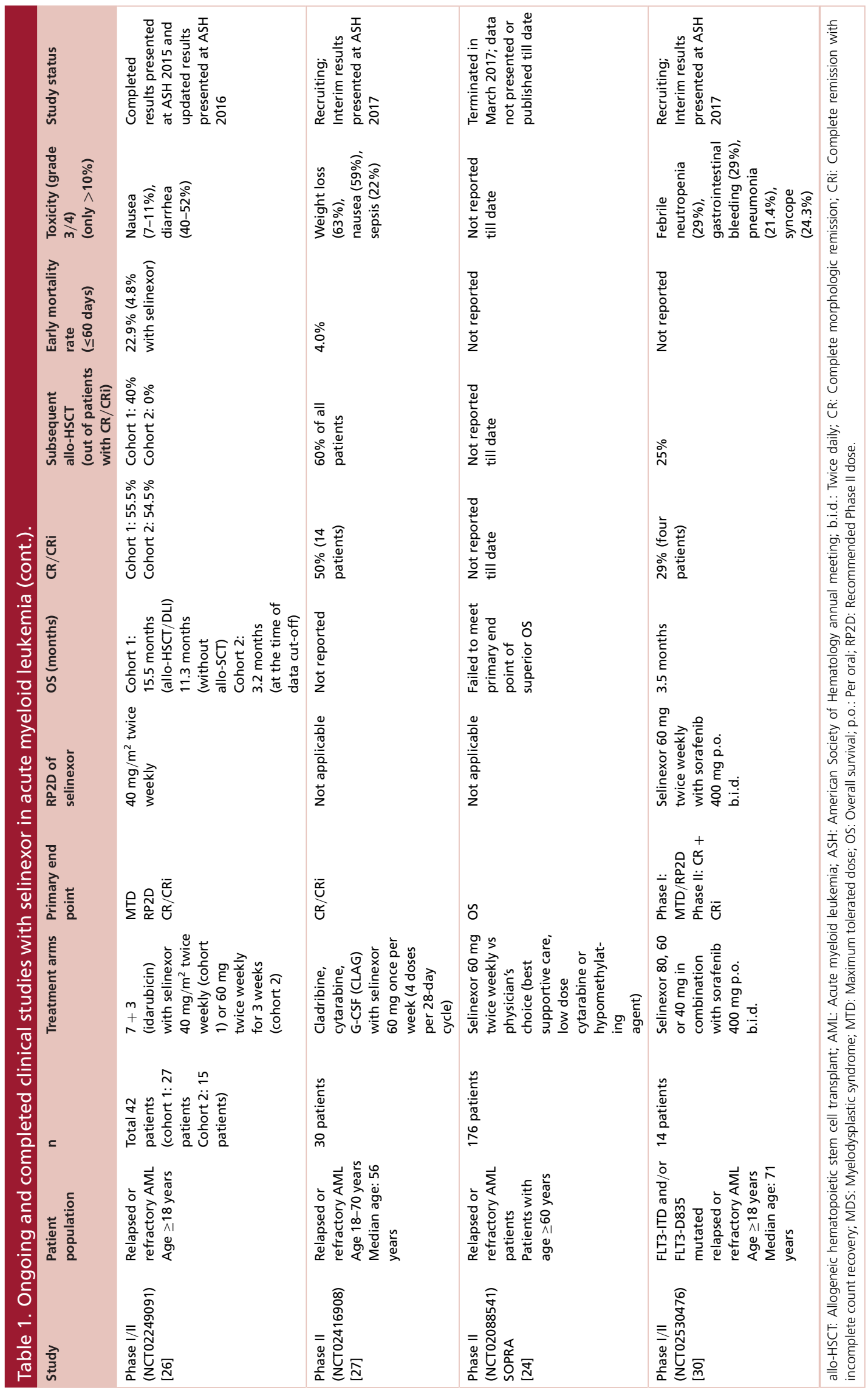


reached and the RP2D of selinexor was $80 \mathrm{mg}$ p.o. twice weekly. At completion of the dose escalation phase, an additional 13 patients were enrolled in an expansion phase at the RP2D. At the time of data cut-off, all 21 patients were evaluable for safety and 18 patients were evaluable for response. The 60 -day mortality was $4.8 \%$. The most common treatment emergent, grade $3 / 4$ nonhematologic adverse events were febrile neutropenia $(76 \%)$, diarrhea (29\%), asymptomatic hyponatremia (32\%) and sepsis (19\%). In terms of the efficacy, ten patients (56\%) achieved a complete remission (CR) or CR with incomplete count recovery (CRi). Among the responders, one (10\%) patient required a second induction. With a median follow-up time of 8.5 months among the responders, $60 \%$ remained in CR/CRi, 80\% were still alive and 50\% had proceeded to allogeneic stem cell transplant (allo-HSCT) [21]. Based on the promising results seen in this Phase I study, a similar Phase II trial is being developed.

\section{Selinexor as a single agent in relapsed/refractory AML}

The efficacy and tolerability of single-agent selinexor was first evaluated by Garzon $e$ t al. in a multicenter Phase I clinical trial that enrolled 95 AML patients (NCT01607892) [23]. Eligible patients included adults with relapsed or refractory AML, those over the age of 60 with treatment-naive AML with antecedent hematological disease or unfavorable cytogenetics, or patients age $\geq 70$ years who were unfit for intensive chemotherapy. The median age of patients enrolled on this trial was 70 years. Selinexor was safe and well tolerated in these older adults. The most common grade $3 / 4$ treatment-related adverse events were thrombocytopenia (20\%), anemia (17\%), fatigue $(14 \%)$ and neutropenia (13\%). The most common nonhematologic toxicities (all grades) were fatigue $(59 \%)$, anorexia $(55 \%)$, nausea $(55 \%)$, diarrhea (40\%), vomiting (38\%), weight loss $(28 \%)$, dysgeusia $(20 \%)$, asymptomatic hyponatremia (22\%) and blurred vision (16\%), however, most were grade $1 / 2$. In spite of being low grade, many of the gastrointestinal-related adverse events, including diarrhea, nausea and anorexia, led to dose interruptions and subsequent dose reductions. The ability of selinexor to penetrate the blood-brain barrier is likely responsible for toxicities such as anorexia, nausea and cerebellar symptoms. Out of 81 evaluable patients, the overall response rate was $14 \%$, including seven patients $(9 \%)$ with CR or CRi. Given the AE profile, pharmacokinetic data and lack of a clear MTD based on dose-limiting toxicities, a flat dosing of $60 \mathrm{mg}$ twice weekly was chosen for the subsequent Phase II study [23].

The randomized Phase II clinical trial Selinexor in Older Patients with Relapsed/Refractory AML (SOPRA) was a multinational study of single-agent selinexor in patients $\geq 60$ years of age with relapsed or refractory AML who were ineligible for intensive chemotherapy. A total of 176 patients were enrolled (NCT02088541). Patients were randomized in a 2:1 fashion to selinexor $60 \mathrm{mg}$ twice a week versus physician's choice (PC). PC included best supportive care, low dose cytarabine or hypomethylating agents. All patients were required to have failed treatment with at least two cycles of an hypomethylating agent prior to enrollment. The primary end point was OS. Results from an interim analysis were announced in March 2017. Although the CR/CRi rate in the selinexor arm was higher than the PC arm (13 vs 3\%, respectively), the early data suggested that this trial was not going to meet its primary end point of improved OS and the study was stopped early due to futility [24].

\section{Selinexor plus chemotherapy in relapsed/refractory AML}

Selinexor was combined with fludarabine and cytarabine in a Phase I study in pediatric patients (age $\leq 24 y e a r s)$ with relapsed or refractory leukemia including AML, acute lymphoblastic leukemia, myelodysplastic syndrome or mixed phenotype acute leukemia (NCT02212561) [25]. Of the 17 patients enrolled on this study, 15 had AML and two had mixed phenotype acute leukemia. Ten patients had previously undergone an allo-HSCT. Selinexor dosing was weight based, and the drug was given twice weekly for 2 weeks prior to beginning induction chemotherapy. Bone marrow biopsies were done on day 15 to assess response to single agent selinexor, prior to beginning fludarabine and cytarabine. Response assessments took place after count recovery. Sixteen patients were evaluable for response to single agent selinexor, and two achieved a CR without evidence of minimal residual disease by flow cytometry. Fifteen patients were evaluable for response to combination therapy, and seven (47\%) achieved a CR/CRi, with five becoming minimal residual disease negative. Inhibition of the XPO1 protein correlated with achievement of response which was demonstrated by at least a twofold decrease in the XPO1 mRNA transcript [25]. A dose of $55 \mathrm{mg} / \mathrm{m}^{2}$ was determined to be tolerable in combination with fludarabine and cytarabine and thus was the RP2D. Two patients developed reversible cerebellar toxicity that was at least possibly related to selinexor. The most common grade 3/4 nonhematologic toxicity was asymptomatic hyponatremia. A Phase II trial is currently being designed in a similar pediatric population to better assess the efficacy of this combination. 
Fiedler $e t$ al. designed a Phase II clinical trial to assess the combination of selinexor with $7+3$ using idarubicin as the choice of anthracycline in a multicenter, single arm study of adult patients with relapsed or refractory AML (NCT02249091) [26]. Two selinexor dose levels were studied in this trial; cohort $1(\mathrm{n}=27) \mathrm{used} 40 \mathrm{mg} / \mathrm{m}^{2}$ twice weekly for 28 days during induction and cohort $2(\mathrm{n}=15)$ used $60 \mathrm{mg}$ twice weekly for 3 weeks during induction. Patients who achieved CR/CRi received consolidation with intermediate or high dose cytarabine in combination with selinexor in a manner similar to induction. Patients who did not proceed with allo-HSCT received selinexor as a maintenance therapy. A total 42 patients were enrolled, and were evaluable for response. Approximately $1 / 3$ of patients had an adverse risk karyotype, and a similar number had relapsed after an allo-HSCT. The CR/CRi rates were 55 and 54\% in cohorts 1 and 2, respectively. The most common selinexor-related AEs in both cohorts were nausea, vomiting and diarrhea. The investigators concluded that the combination of selinexor and $7+3$ has an acceptable tolerability profile with a promising efficacy in a heavily pretreated AML population.

A combination of selinexor with CLAG induction (cladribine $5 \mathrm{mg} / \mathrm{m}^{2} / \mathrm{d}$ on days $4-8$, cytarabine $2000 \mathrm{mg} / \mathrm{m}^{2} / \mathrm{d}$ on days $4-8$ and granulocyte colony-stimulating factor $300 \mathrm{mcg} / \mathrm{d}$ on days 3-8) was also studied in a single arm, Phase II trial for adult patients with relapsed or refractory AML (NCT02416908) [27]. A total of 30 patients were enrolled with median age of 56 years. The majority of patients $(63 \%)$ were in their first relapse, while $33 \%$ were refractory to their initial induction chemotherapy. The CR/CRi rate was 50\%, and the early mortality (defined as death within 30 days of treatment initiation) was only $4 \%$. Most common nonhematologic treatment emergent adverse events included anorexia with weight loss in $63 \%$ and nausea in $59 \%$. The incidence of diarrhea was not reported. Of the first 25 patients treated on this trial, $60 \%$ were able to proceed to allo-HSCT [27].

Another Phase I clinical trial combined selinexor with high-dose cytarabine (HiDAC) and mitoxantrone and enrolled 12 adult patients with newly diagnosed or relapse/refractory AML (NCT02299518). The median age was 61 years, and $67 \%$ were previously untreated. Selinexor was given at either $60 \mathrm{mg}$ or $80 \mathrm{mg}$ on days 2, 4, 9 and 11 of induction. Ten patients were evaluable for safety and efficacy, and five (50\%) achieved a CR/CRi. No responses were seen in the four relapsed/refractory patients. The most common adverse events were myelosuppression and febrile neutropenia, and the median time to count recovery was 38 days [22].

\section{Selinexor in FLT3-mutated relapsed or refractory AML}

Data from Zhang et al. using murine models and primary AML patient samples demonstrated that selinexor has antileukemic activity in FLT3-mutant AML, including internal tandem duplication (ITD), tyrosine kinase domain (TKD) and compound mutations [28]. This antileukemic effect occurs via modulation of the cdk inhibitor, p27 and the antiapoptotic protein, MCL-1. Moreover, when combined with sorafenib, a multikinase inhibitor with activity against FLT3-ITD, the antileukemic effects were more pronounced with lower phosphorylation of FLT3 and a reduction in activation of downstream pathways. The combination of selinexor- and sorafenib-induced differentiation of the leukemic cells harbors the FLT3 mutation [28,29]. To this end, Daver $e$ al. designed a Phase I/II clinical trial of selinexor in combination with sorafenib for FLT3-mutated relapsed or refractory AML patients (NCT02530476) [30]. The RP2D of selinexor was $60 \mathrm{mg}$ twice weekly in combination with sorafenib $400 \mathrm{mg}$ twice daily, and early responses have been encouraging. Of the first 14 patients enrolled, four (28\%) achieved CR/CRi. An additional two (14\%) patients had $\geq 50 \%$ blast reduction. All six of these patients had received prior FLT3 inhibitor treatment. The most common grade $3 / 4$ adverse events included bleeding $(35 \%)$ and febrile neutropenia (28.6\%). This study is continuing to enroll patients at this time [30].

\section{Selinexor as a post-transplant maintenance strategy}

Selinexor is also being explored as a post allo-HSCT maintenance strategy in a Phase I trial of patients with intermediate and high-risk AML and myelodysplastic syndrome (NCT02485535) [31]. Patients were enrolled 60100 days after allo-HSCT. A total of nine patients were accrued between January 2016 and March 2017. Eligible patients had no evidence of graft-versus-host disease. The primary end point was the MTD of selinexor, which was $60 \mathrm{mg}$ once a week. With a median follow-up of 359 days from transplant, the median duration of remission is 199 days (59-542). Two patients (22\%) have relapsed thus far. One patient relapsed after two cycles and one after four cycles of treatment. Nonetheless, these data are still maturing and this trial is continuing to accrue patients in the expansion cohort to better characterize the toxicity profile and clinical efficacy [31]. 
Selinexor in malignancies other than AML

Apart from AML, selinexor is currently in early phase clinical trials in other malignancies. The efficacy of selinexor was assessed as monotherapy in patients with metastatic castrate-resistant prostate cancer that is refractory to antiandrogen therapies including abiraterone, enzalutamide, apalutamide or other investigational agents [32]. This Phase II study failed to achieve its primary end point of PFS and was terminated early after enrollment of 14 out of the planned 54 patients. A significant proportion of the patients $(36 \%)$ developed grade $3 / 4$ treatment-related serious adverse events including anorexia, nausea and fatigue. Efficacy data were not convincing to support further clinical investigation in this patient population. Vogl et al. assessed the efficacy of selinexor in combination with dexamethasone in relapsed or refractory multiple myeloma and reported encouraging clinical activity [33]. The Phase II clinical trial evaluated selinexor $80 \mathrm{mg}$ and dexamethasone $20 \mathrm{mg}$ given weekly in relapsed/refractory multiple myeloma patients resulting in an overall response rate of $21 \%$. Moreover, in patients with high-risk cytogenetics $(\mathrm{t}[4 ; 14]$ and $\operatorname{del}[17 \mathrm{p}])$, the ORR was $35 \%$. The median duration of response was 5 months with $65 \%$ of the responding patients still alive at 1 year. The most common grade 3 adverse events included thrombocytopenia, anemia, neutropenia, hyponatremia and fatigue. Such studies indicate varying clinical efficacy and tolerance across different malignancies, however the side effect profile is consistent in these various patient populations.

\section{Second generation SINE KPT-8602}

KPT-8602 is a highly potent, second-generation SINE with robust in vitro activity against AML blasts and leukemiainitiating cells as demonstrated by Etchin et al. [34]. This is an oral compound with similar pharmacokinetic properties to selinexor, however, it has a markedly reduced ability to penetrate the blood-brain barrier (30-fold less). Given that the dose-limiting toxicity of selinexor is resultant of CNS-mediated anorexia with weight loss and malaise, this new compound appears very promising [35]. Compared with selinexor, KPT-8602 exerted superior efficacy with preferential lethality against leukemia-initiating cells in the patient-derived xenograft murine models with minimal effects on the normal hematopoietic stem and progenitor cells. Moreover, the tolerability of KPT-8602 was better in patient-derived xenograft mouse models, likely due to the minimal CNS penetration as compared with selinexor [34]. Currently KPT-8602 is being investigated in multiple myeloma in Phase I/II clinical trial however it is not yet being studied in AML.

\section{Future perspective}

Deeper understanding of the biology of AML, and evolving knowledge about the mechanisms of resistance, has unlocked the doors to devising novel therapeutic approaches which may potentially lead to improved, individualized management. By forcing nuclear retention of signal-containing cargo proteins, including nearly all known TSPs and growth regulators, this novel approach of nuclear transport inhibition may have the ability to overcome resistance to traditional chemotherapy. Although selinexor is less likely to be utilized as a monotherapy, given the low remission rates in this setting, it has shown early potential to overcome treatment resistance when combined with the conventional cytotoxic chemotherapy. Moreover, given its ability to force nuclear retention of the XPO1 cargo protein NPM1, which is involved in ribosomal assembly and trafficking as well as DNA repair, this perhaps serves as a rational therapeutic strategy for NPM1 ${ }^{\text {mut }}$ AML $[36,37]$. Mutations in NPM1 create a biding site for XPO1 leading to its cytoplasmic translocation and functional deactivation, therefore targeting these leukemia cells with a SINE is a sensible approach [38].

\section{Conclusion}

Altering the nuclear transport of various proteins, including many TSPs such as $\mathrm{p} 53$, NPM1 and NFK $\beta$, plays a pivotal role in proliferation and propagation of leukemia stem cells and AML blasts, enabling resistance to traditional chemotherapeutic approaches. SINE provide a promising therapeutic strategy with favorable, early clinical efficacy, specifically when used in combination with traditional chemotherapeutic agents. Further identification of specific AML populations, based on cytogenetic and molecular characteristics, is necessary in order to determine which patients are most likely to benefit from this enticing therapeutic option.

Financial \& competing interests disclosure

KL Sweet received honorarium and research funding from Karyopharm in 2016. The authors have no other relevant affiliations or financial involvement with any organization or entity with a financial interest in or financial conflict with the subject matter or materials discussed in the manuscript apart from those disclosed.

No writing assistance was utilized in the production of this manuscript. 


\section{Open access}

This work is licensed under the Attribution-NonCommercial-NoDerivatives 4.0 Unported License. To view a copy of this license, visit http://creativecommons.org/licenses/by-nc-nd/4.0/

\section{References}

Papers of special note have been highlighted as: • of interest; $\bullet \bullet$ of considerable interest

1. Garg M, Nagata Y, Kanojia D et al. Profiling of somatic mutations in acute myeloid leukemia with FLT3-ITD at diagnosis and relapse. Blood 126(22), 2491-2501 (2015).

2. Bishop JF, Lowenthal RM, Joshua D et al. Etoposide in acute nonlymphocytic leukemia. Australian Leukemia Study Group. Blood 75(1), 27-32 (1990).

3. Holowiecki J, Grosicki S, Giebel S et al. Cladribine, but not fludarabine, added to daunorubicin and cytarabine during induction prolongs survival of patients with acute myeloid leukemia: a multicenter, randomized Phase III study. J. Clin. Oncol. 30(20), 2441-2448 (2012).

4. Lowenberg B, Ossenkoppele GJ, Van Putten W et al. High-dose daunorubicin in older patients with acute myeloid leukemia. N. Engl. J. Med. 361(13), 1235-1248 (2009).

5. Fernandez HF, Sun Z, Yao X et al. Anthracycline dose intensification in acute myeloid leukemia. N. Engl. J. Med. 361(13), 1249-1259 (2009).

6. Lancet JE, Uy GL, Cortes JE et al. Final results of a Phase III randomized trial of CPX-351 versus $7+3$ in older patients with newly diagnosed high risk (secondary) AML. J. Clin. Oncol. 34(Suppl. 15), 7000 (2016).

7. Stone RM, Mandrekar SJ, Sanford BL et al. Midostaurin plus chemotherapy for acute myeloid leukemia with a FLT3 mutation. N. Engl. J. Med. 377(5), 454-464 (2017).

8. Itzykson R, Thepot S, Berthon C et al. Azacitidine for the treatment of relapsed and refractory AML in older patients. Leuk. Res. 39(2), $124-130$ (2015).

9. Dombret H, Seymour JF, Butrym A et al. International Phase III study of azacitidine vs conventional care regimens in older patients with newly diagnosed AML with > 30\% blasts. Blood 126(3), 291-299 (2015).

10. Turner JG, Dawson J, Sullivan DM. Nuclear export of proteins and drug resistance in cancer. Biochem. Pharmacol. 83(8), 1021-1032 (2012).

11. Shinmura K, Tarapore P, Tokuyama Y, George KR, Fukasawa K. Characterization of centrosomal association of nucleophosmin/B23 linked to Crm1 activity. FEBS Lett. 579(29), 6621-6634 (2005).

12. Kojima K, Kornblau SM, Ruvolo V et al. Prognostic impact and targeting of CRM1 in acute myeloid leukemia. Blood 121(20), 4166-4174 (2013).

-• First study that links expression of XPO1 to survival and analyzes prognostic implication based on cytogenetic and molecular findings.

13. Whitman SP, Ruppert AS, Radmacher MD et al. FLT3 D835/I836 mutations are associated with poor disease-free survival and a distinct gene-expression signature among younger adults with de novo cytogenetically normal acute myeloid leukemia lacking FLT3 internal tandem duplications. Blood 111(3), 1552-1559 (2008).

14. Yanada M, Matsuo K, Suzuki T, Kiyoi H, Naoe T. Prognostic significance of FLT3 internal tandem duplication and tyrosine kinase domain mutations for acute myeloid leukemia: a meta-analysis. Leukemia 19(8), 1345-1349 (2005).

15. Godley LA. A drug that stops traffic at the nuclear border. Blood 120(9), 1759-1760 (2012).

16. Ranganathan P, Yu X, Na C et al. Preclinical activity of a novel CRM1 inhibitor in acute myeloid leukemia. Blood 120(9), 1765-1773 (2012).

-. Describes the mode of action of selinexor in vitro and forms the rationale for clinical studies.

17. Turner JG, Engel R, Derderian JA, Jove R, Sullivan DM. Human topoisomerase IIalpha nuclear export is mediated by two CRM-1-dependent nuclear export signals. J. Cell Sci. 117(Pt 14), 3061-3071 (2004).

18. Mirski SE, Cole SP. Cytoplasmic localization of a mutant M(r) 160,000 topoisomerase II alpha is associated with the loss of putative bipartite nuclear localization signals in a drug-resistant human lung cancer cell line. Cancer Res. 55(10), 2129-2134 (1995).

19. Mutka SC, Yang WQ, Dong SD et al. Identification of nuclear export inhibitors with potent anticancer activity in vivo. Cancer Res. 69(2), 510-517 (2009).

20. Ranganathan P, Kashyap T, Yu X et al. XPO1 inhibition using selinexor synergizes with chemotherapy in acute myeloid leukemia by targeting DNA repair and restoring topoisomerase iialpha to the nucleus. Clin. Cancer Res. 22(24), 6142-6152 (2016).

- Serves as a preclinical rational for combining selinexor with chemotherapy in acute myeloid leukemia (AML).

21. Sweet KL, Komrokji RS, Padron E et al. A Phase I study of selinexor in combination with daunorubicin and cytarabine in patients with newly diagnosed poor-risk acute myeloid leukemia. Blood 128(22), 4040 (2016).

- Phase I study assessed tolerance of selinexor with $7+3$ chemotherapy in de novo AML and demonstrated tolerable profile. 
22. Wang AY, Weiner HL, Green M et al. Combination of selinexor with high-dose cytarabine (HiDAC) and mitoxantrone (Mito) for remission induction in acute myeloid leukemia (AML) is feasible and tolerable. Blood 128(22), 212 (2016).

23. Garzon R, Savona M, Baz R et al. A Phase I clinical trial of single-agent selinexor in acute myeloid leukemia. Blood 129(24), 3165-3174 (2017).

-. This is the first study that assessed efficacy of selinexor as monotherapy in AML.

24. Karyopharm Press Release. Karyopharm annouces results from interim analysis of Phase II OPRA study evaluating selinexor in relapsed/refractory acute myeloid leukemia. http://investors.karyopharm.com/releasedetail.cfm?ReleaseID=1015594.

25. Alexander TB, Lacayo NJ, Choi JK, Ribeiro RC, Pui CH, Rubnitz JE. Phase I study of selinexor, a selective inhibitor of nuclear export, in combination with fludarabine and cytarabine, in pediatric relapsed or refractory acute leukemia. J. Clin. Oncol. 34(34), 4094-4101 (2016).

26. Fiedler W, Heuser M, Chromik J et al. Phase II results of Ara-C and idarubicin in combination with the selective inhibitor of nuclear export (SINE) compound selinexor (KPT-330) in patients with relapsed or refractory AML. Blood 128(22), 341 (2016).

- Highlights efficacy of the combination of selinexor with $7+3$ in relapsed/refractory AML.

27. Uy GL, Rettig MP, Fletcher T et al. Selinexor in combination with cladribine, cytarabine and G-CSF for relapsed or refractory AML. Blood 130(Suppl. 1), 816 (2017).

28. Zhang W, Ishizawa J, Mu H, Daver N, Ruvolo V, Andreeff M. Combinatorial targeting of XPO1 and FLT3-ITD exerts synergistic antitumor effects in FLT3-mutated acute myeloid leukemias. Blood 126(23), 1266 (2015).

29. Yamatani K, Tabe Y, Sekihara K et al. XPO1 inhibition targets transcriptional vulnerability of FLT3-ITD+D835 double mutant AML through p 53 accumulation and inhibition of oncogenic transcription factors: lesson learned from cage sequencing of primary AML cells. Blood 130(Suppl. 1), 1244 (2017).

30. Daver N, Assi R, Garcia-Manero G et al. A Phase I/II study of selinexor (SEL) with sorafenib in patients (pts) with relapsed and/or refractory (R/R) FLT3 mutated acute myeloid leukemia (AML). Blood 130(Suppl. 1), 1344 (2017).

31. Liu H, Artz A, Larson RA, Kosuri S, Stock W, Bishop MR. Selinexor maintenance is feasible and tolerable after allogeneic stem cell transplant (allo-SCT) for patients with acute myeloid leukemia (AML) and myelodysplastic syndrome (MDS). Blood 130(Suppl. 1), 3312 (2017).

32. Wei XX, Siegel AP, Aggarwal R et al. A Phase II trial of selinexor, an oral selective inhibitor of nuclear export compound, in abirateroneand/or enzalutamide-refractory metastatic castration-resistant prostate cancer. Oncologist doi:10.1634/theoncologist.2017-0624 (2018) (Epub ahead of print).

33. Vogl DT, Dingli D, Cornell RF et al. Selective inhibition of nuclear export with oral selinexor for treatment of relapsed or refractory multiple myeloma. J. Clin. Oncol. 36(9), 859-866 (2018).

34. Etchin J, Berezovskaya A, Conway AS et al. KPT-8602, a second-generation inhibitor of XPO1-mediated nuclear export, is well tolerated and highly active against AML blasts and leukemia-initiating cells. Leukemia 31(1), 143-150 (2017).

-. Discusses second-generation selective inhibitors of nuclear export with an improved efficacy and toxicity profile in a preclinical study.

35. Hing ZA, Fung HY, Ranganathan P et al. Next-generation XPO1 inhibitor shows improved efficacy and in vivo tolerability in hematological malignancies. Leukemia 30(12), 2364-2372 (2016).

36. Falini B, Martelli MP, Bolli N et al. Immunohistochemistry predicts nucleophosmin (NPM) mutations in acute myeloid leukemia. Blood 108(6), 1999-2005 (2006).

37. Falini B, Mecucci C, Tiacci E et al. Cytoplasmic nucleophosmin in acute myelogenous leukemia with a normal karyotype. N. Engl. J. Med. 352(3), 254-266 (2005).

38. Falini B, Bolli N, Liso A et al. Altered nucleophosmin transport in acute myeloid leukaemia with mutated NPM1: molecular basis and clinical implications. Leukemia 23(10), 1731-1743 (2009).

- Explores use of selinexor as a 'targeted therapy' for a specific subpopulation of AML based on the mechanism of selective inhibitors of nuclear export. 